Joachim Ballweg

\title{
Eine Dynamische Zeitlogik für das Deutsche
}

In diesem Beitrag soll eine dynamische Zeitlogik skizziert werden, die eine Analyse der deutschen Tempora über den Satzrahmen hinaus ermöglicht. Basis dieser Darstellung soll eine Reichenbachianische Analyse der Deutschen Tempora sein. ${ }^{1}$

Zunächst wird eine Sprache ADETAL angegeben (Adverbial erweiterte temporale Aussagenlogik). Für diese Sprache wird eine Semantik im Stile Reichenbachs formuliert. ${ }^{2}$ Diese erweist sich als adäquat zur Beschreibung einzelner Sätze. Um auch noch die temporale Struktur von Sätzen zu beschreiben, werden wir eine dynamische Perspektive entwickeln, indem wir Ideen aus der Dynamischen Prädikatenlogik von Groenendijk/Stokhof ${ }^{3}$ aufgreifen. Schließlich soll das System bei der Analyse eines Textausschnitts auf die Probe gestellt werden.

1 Eine ausfuhrliche Darstellung findet sich in Ballweg 1988. Die hier skizzierten Ideen sind im Reichenbachianischen Teil stark von den Arbeiten von L. Aqvist, Chr. Rohrer und F. Guenthner beeinfluBt, im dynamischen Teil von den DRT-Analysen franzosischer Tempora von H. Kamp and Chr. Rohrer und bei der Behandlung der Adverbialia von Smith 1977. Für Diskussionen und Hinweise danke ich meinen Kolleginnen und Kollegen am IdS Mannheim Ursula Brauße, Ursula Hoberg, Renate Pasch, Angelika Storrer, Gisela Zifonun, Bruno Strecker und Helmut Frosch. Weiter habe ich zu danken den Teilnehmern meiner Seminare in Stuttgart, SS 1993 und WS 1995/96, sowie des Linguistenseminars in Kyoto 1995; Günther Grewendorf und Manfred Bierwisch danke ich dafuir, daß sie mir im Sommer $1993 \mathrm{Ge}$ legenheit geboten haben, eine vorlăufige Fassung in Frankfurt bzw. Berlin zur Diskussion zu stellen. Neben den beiden genannten waren dabei vor allem Jochen Zeller in Frankfurt, Reinhard Blutner und Manfred Krifka in Berlin anregende Diskussionspartner.

2 Siehe Reichenbach 1947.

3 Siehe Groenendijk/Stokhof 1991. 
1 Die Sprache ADETAL: Syntax

1.1 Vokabular und Formeldefinition

Satzbuchstaben:

Im Vorgriff auf eine mögliche prädikatenlogische Erweiterung führen wir eine Unterscheidung von telischen und atelischen Sätzen ein. ${ }^{4}$

$\mathrm{SB}=$ SBTEL $\cup$ SBATEL

SBTEL $=\left\{a\left|, a^{1}\right|, \ldots, z \mid\right\}$

SBATEL $=\left\{a, a^{1}, \ldots, z^{n}\right\}$

Junktoren: $\mathbf{J}=\{\neg\}$

Tempusoperatoren:

Die Tempusoperatoren erscheinen in zwei Untermengen aufgeteilt, wobei TO 1 die einfachen, finiten Tempora umfaßt, TO 2 das Perfekt. Dadurch wird eine kompositionale Analyse der zusammengesetzten Zeiten möglich:

$\mathrm{TO}=\mathrm{TO} 1 \cup \mathrm{TO} 2$

$\mathrm{TO} 1=\{$ Pres, Pret, Fut $\}$

$\mathrm{TO2}=\{$ Perf $\}$

Temporaladverbialia:

$\mathrm{TADV}=\quad$ gestern, heute, da, dann, ... $\}$

Temporaladverbialformatoren:

TAFO $=\quad\{$ als, bis, $\ldots\}$

Hilfszeichen der syntaktischen Gliederung:

$\mathrm{HZ}=\{()$,

4 Siehe dazu ausführlich Krifka 1989. 


\subsection{Formeldefinition}

WFF ist die Menge der wohlgeformten Formeln von ADETAL: Es gibt drei verschiedene Formeltypen, nämlich untemporalisierte $\left(\mathrm{WFF}^{0}\right)$, temporalisierte $\left(\mathrm{WFF}^{\mathrm{t}}\right)$ und perfektivierte $\left(\mathrm{WFF}^{12}\right)$ :

$\mathrm{WFF}=\mathrm{WFF}^{0} \cup \mathrm{WFF}^{11} \cup \mathrm{WFF}^{12}$

- Ist $\alpha \in \mathrm{SB}$, so ist $\alpha \in \mathrm{WFF}$.

- Ist $\beta \in \mathrm{WFF}^{0}, \alpha \in \mathrm{TO} 2$, so ist $\alpha(\beta) \in \mathrm{WFF}^{12}$.

- Ist $\alpha \in \mathrm{TO} 1, \beta \in \mathrm{WFF}^{10} \cup \mathrm{WFF}^{12}$, so ist $\alpha(\beta) \in \mathrm{WFF}^{11}$.

- Ist $\alpha \in \mathrm{TAFO}, \beta \in \mathrm{WFF}^{11} \cup \mathrm{WFF}^{12}$, so ist $\alpha(\beta) \in \mathrm{TADV}$.

Seien $\beta, \gamma$ Formeln eines beliebigen, aber gleichen Typs, dann gilt:

- Ist $\alpha \in$ TADV, so ist $\alpha(\beta)$ eine WFF vom selben Typ wie $\beta$.

- Ist $\alpha=\neg(\beta)$, so ist $\alpha$ eine WFF vom selben Typ wie $\beta$.

- Ist $\alpha=(\beta \wedge \gamma)$, so ist $\alpha$ eine WFF vom selben Typ wie $\beta$ bzw. $\gamma$.

Nicht sonst ist aus WFF.

\section{$1.3 \quad$ Kommentare}

Syntaktisch gesehen ist ADETAL eine Sprache der Aussagenlogik, die durch Tempusoperatoren und Operatoren zur Wiedergabe von temporalen Adverbialia erweitert ist. Unorthodox ist die Aufteilung der Elementarsätze in die zwei Subtypen telisch und atelisch sowie die Aufspaltung der Tempusoperatoren in zwei Gruppen, wodurch die Formeln von Adetal in sechs Typen aufgespalten sind, nämlich telische bzw. atelische untemporalisierte (Superskript 0), telische bzw. atelische untemporalisierte (Superskript $\mathrm{t} 1$ ) und telische bzw. atelische perfektivierte (Superskript t2).

Die Idee hinter dieser Konstruktion ist, daß untemporalisierte Formeln für tempuslose Satzradikale (mit dem Verb im Infinitiv) stehen, perfektivierte Formeln für Satzradikale mit dem Verb im Infinitiv Perfekt, und daß durch Anwendung der TO 1 - Operatoren daraus temporalisierte Formeln werden, die Sätzen mit finiten Verben entsprechen. Dadurch wird eine kompositionale Analyse der zusammengesetzten Tempora des Deutschen erreicht: 
p

Sherlock Holmes Dr. Moriarty jagen.

Perf(p)

Sherlock Holmes Dr. Moriarty gejagt haben.

$\operatorname{Pres}(\mathrm{p})$

Pret (p)

Sherlock Holmes jagt Dr. Moriarty.

Fut (p)

Sherlock Holmes jagte Dr. Moriarty.

$\operatorname{Pres}(\operatorname{Perf}(\mathrm{p}))$

Sherlock Holmes wird Dr. Moriarty jagen.

$\operatorname{Pret}(\operatorname{Perf}(\mathrm{p}))$

Sherlock Holmes hat Dr. Moriarty gejagt.

Fut(Perf(p))

Sherlock Holmes hatte Dr. Moriarty gejagt.

Sherlock Holmes wird Dr. Moriarty gejagt haben.

Außerdem ist zu beachten, daß es in unserer Sprache Skopusambiguitäten bei Temporaladverbialia gibt, so hat

1812 hat Napoleon halb Europa erobert

drei Übersetzungen, nämlich: 1812(Pret(Perf(p))), Pret(1812(Perf(p))), und Pret(Perf(1812(p)))! Dies hat natürlich Folgen für die Konstruktion der Semantik.

2 Die Sprache ADETAL: Semantik

2.1 Reichenbachianische Interpretation von Adetal

Ein Reichenbachianisches Modell $\mathfrak{R}$ für die Interpretation von Adetal ist eine Struktur der folgenden Art:

$\Re=((\mathrm{T} ., \angle),(\mathrm{T},<),(\mathrm{t} 0, \mathrm{tb}, \mathrm{te}), f, g)$

Wir erläutern die einzelnen Komponenten von $\mathfrak{N}$ wie folgt: 
(T., $\angle$ ) ist der Zeitstrahl; dabei ist $\mathrm{T}$. die Menge der Zeitpunkte, und $\angle$ ist eine konnexe, transitive irreflexive und dichte Ordnung auf $T$., das heißt, furr alle t., t.', t." $\in \mathrm{T}$. gilt:

- entweder t. $\angle \mathrm{t}$.' oder t' $\angle \mathrm{t}$ oder $\mathrm{t}$. $=\mathrm{t}$.' (Die Ordnung ist konnex.);

- gilt $\mathrm{t}$. $\angle \mathrm{t}$.' und t.' $\angle \mathrm{t}$.", so gilt auch t. $\angle \mathrm{t}$." (Die Ordnung ist transitiv.);

- nicht: $t$. $\angle \mathrm{t}$. (Die Ordnung ist irreflexiv.);

- gilt t. $\angle$ t.', so gibt es ein t.", so daß t. $\angle$ t." $\angle$ t.' (Die Ordnung ist dicht.).

Außerdem gibt es zu jedem t. ein t., so daß t. $\angle \mathrm{t}$.' und ein $t$.", so daß t." $\angle \mathrm{t}$. ( $T$. ist nach beiden Seiten unendlich.).

-( $\mathrm{T},<)$ : $-\mathrm{T}$ ist die Menge der Zeitintervalle, die folgendermaßen definiert ist: furr alle $X$, so daß: $T$. $\supseteq X$ gilt: Wenn $X$ bezüglich der Ordnung $\angle$ konvex ist, so gilt $\mathrm{X} \in \mathrm{T}$.

NB: Eine Untermenge $U$ einer Menge $M$ ist konvex bezüglich einer Ordnung $o$, wenn furr alle $x, x^{\prime} \in U, x^{\prime \prime} \in M$ gilt: aus $x$ o $x^{\prime}, x$ o $x^{\prime \prime}$ und $x^{\prime \prime} \circ x^{\prime}$ folgt: $x^{\prime \prime} \in U$.

Gilt $t \supset t^{\prime}$, so nennen wir t' (echtes) Subintervall von $t$ und $t$ Superintervall von t'. Man beachte, daß T auch T (unecht) enthält, sowie die leere Menge und alle Intervalle, die nur einen Zeitpunkt enthalten.

- < ist eine Ordnung auf $T$, so daß furr alle $t, t^{\prime} \in T$ gilt: gibt es ein $t . \in t$, so daß für alle $t$.' $\in$ t' gilt: $t$. $\angle \mathrm{t}$.', so $\mathrm{t}<\mathrm{t}$ '. Die Ordnung $<$ ist von $\angle$ und übernimmt alle Eigenschaften von $\angle$ bis auf die Konnexität, ist also transitiv, irreflexiv und dicht.

Außerdem können wir eine reflexive Relation * für Überlappung von Intervallen einführen: $t^{*} t^{\prime} g d w . t \cap t^{\prime} \neq \varnothing$. In $T \backslash S$ ist ${ }^{*}$ zusätzlich dicht.

Aus < können wir eine transitive, irreflexive und dichte Relation $\mathrm{h}$ für , vollständig vor" ableiten: für alle $t, t^{\prime} \in T$ gilt: $t h t^{\prime} g d w . t<t^{\prime}$ und $t \cap t^{\prime}=\varnothing^{5}$

t0, tr und te sind drei ausgezeichnete Intervalle, das Sprech-, das Referenz- und das Evaluationsintervall. ${ }^{6}$

5 Zum Zusammenhang von Intervall- und Punktstrukturen ausführlich Kamp 1979, sowie van Benthem.

6 Diese "Drei-Intervall-Theorie" geht letztlich auf Reichenbach 1947 zurück: man vergleiche auch Åqvist 1976, Åqvist/Guenthner 1978. 
$f$ ist eine Funktion von $\mathrm{SB} \times \mathrm{T}$ nach $\{0,1\}$, so daß für alle $\alpha \in \mathrm{SB}$, für alle $\mathrm{t}, \mathrm{t}$, $\mathrm{t}^{\prime \prime} \in \mathrm{T}$ gilt:

- Wenn $\alpha \in$ SBATEL, dann ist $f(\alpha, \mathrm{t})=1 \mathrm{gdw}$. für mindestens ein $\mathrm{t}$ " * $\mathrm{t}$ gilt: $f\left(\alpha, \mathrm{t}^{\prime \prime}\right)=1 ;$ wenn $f(\alpha, \mathrm{t})=1$ und wenn $\mathrm{t}^{\prime} \subseteq \mathrm{t}$, dann $f\left(\alpha, \mathrm{t}^{\prime}\right)=1$.

- wenn $\alpha \in$ SBTEL, dann ist $f(\alpha, t)=1$ gdw. für mindestens ein t' $\subseteq \mathrm{t}$ gilt: $f\left(\alpha, \mathrm{t}^{\prime \prime}\right)=1$; wenn $f(\alpha, \mathrm{t})=1$ und $\mathrm{t}^{\prime} \subset \mathrm{t} \subset \mathrm{t}^{\prime \prime}, \operatorname{dann} f\left(\alpha, \mathrm{t}^{\prime}\right)=f\left(\alpha, \mathrm{t}^{\prime \prime}\right)=0$; wenn $t \in S$, so $f(\alpha, t)=0$.

Diese Klauseln stellen sicher, daß ein Elementarsatz $\alpha$ dann als wahr bewertet wird bezüglich einem Intervall $t$, wenn $t$ sich mit einem Wahrheitsintervall von $\alpha$ überlappt. Außerdem wird festgelegt, daß Wahrheitsintervalle von atelischen Satzradikalen homogen sind, so sind z.B. alle Teilintervalle eines Wahrheitsintervalles des atelischen Satzradikals

\section{Es regnen}

selbst auch Wahrheitsintervalle dieses Satzradikals.

Die Klausel für telische Satzradikale garantiert schließlich noch, daß Wahrheitsintervalle von telischen Satzradikalen inhomogen sind; so sind z.B. alle Subintervalle eines telischen Satzradikals

Herbert das Matterhorn besteigen

keine Wahrheitsintervalle davon, und auch Superintervalle von Wahrheitsintervallen von telischen Sätzen sind keine; außerdem werden an Intervallen aus S telische Sätze immer mit 0 bewertet. Die Idee dabei ist natürlich, daß inhomogene Intervalle ausgedehnt sein müssen. ${ }^{7}$

7 Vielleicht wäre es sinnvoll, für solche Sätze, wie Löbner 1988 in Fußnote 15 vorschlägt, nur offene Intervalle als Wahrheitsintervalle zuzulassen. Verben wie z. B. einschlafen könnte man dann so beschreiben, daß die Zustandsänderung in dem offenen Intervall stattfindet, das durch den letzten Punkt des Wahrheitsintervalls des Vorzustandes und den ersten Punkt des Wahrheitsintervalls des Nachzustandes aufgespannt wird,

$\begin{array}{lll}\text { wach sein ta] einschlafen } & \text { tb[ schlafen }\end{array}$

vgl. Löbner 1988, S. 186. 
- $g$ ist eine Funktion von WFF $\times \mathrm{T} \times \mathrm{T} \times \mathrm{T}$ nach $\{0,1\}$, so daß für alle $\alpha, \beta, \gamma \in$ WFF, für alle $\mathrm{t} 1, \mathrm{t} 2, \mathrm{t} 3 \in \mathrm{T}$ gilt:

- Ist $\alpha \in \mathrm{SB}$, so ist $g(\alpha, \mathrm{t} 1, \mathrm{t} 2, \mathrm{t} 3)=1 \mathrm{gdw} . f(\alpha, \mathrm{t} 3)=1$.

- Ist $\alpha=\neg(\beta)$, so ist $g(\alpha, \mathrm{t} 1, \mathrm{t} 2, \mathrm{t} 3)=1 \mathrm{gdw} . g(\beta, \mathrm{t} 1, \mathrm{t} 2, \mathrm{t} 3)=0$.

- Ist $\alpha=(\beta \wedge \gamma)$, so ist $g(\alpha, \mathrm{t} 1, \mathrm{t} 2, \mathrm{t} 3)=1 \mathrm{gdw} . g(\beta, \mathrm{t} 1, \mathrm{t} 2, \mathrm{t} 3)=g(\gamma, \mathrm{t} 1, \mathrm{t} 2, \mathrm{t} 3)$ $=1$.

\section{Tempus-Operatoren}

Generell werden Tempusoperatoren aufgefaßt als Spezifikation der Relation zwischen Sprech- und Betrachtzeit. Für alle Tempora kann man dann unter Vernachlässigung der telisch-atelisch Unterscheidung die Überlappung von Betracht- und Evaluationszeit festlegen. ${ }^{8}$ Ein Modell $\mathfrak{R}$ nennen wir geeignet für die Interpretation eines Tempus, wenn die Relation zwischen Betracht- und Evaluationszeit der Festlegung für die Semantik des entsprechenden Tempusoperators entspricht. Im Folgenden werden wir, jeweils nach einigen intuitiven Bemerkungen, die Interpretationsklauseln für die einzelnen Tempusoperatoren angeben.

\section{Präsens}

Die erste Interpretationsidee ist natürlich, das Präsens dadurch zu charakterisieren, daß man die Sprech- und die Betrachtzeit gleichsetzt. Damit ergäbe sich dann automatisch eine Überlappung von Sprech- und Evaluationszeit. Betrachtet man jedoch Beispiele wie

(1) Zwei mal zwei ist vier.

(2) Morgen kommt der Kaminfeger.

(3) 1618 bricht der dreißigjährige Krieg aus.

so erkennt man, daß diese Interpretation inadäquat wäre, da das deutsche Präsens es erlaubt, daß die Betrachtzeit nach (wie in 2) oder vor (wie in 3) der Sprechzeit liegt. Unsere Analyse für das Präsens trägt dem Rechnung, indem überhaupt keine Relation zwischen Sprech- und Betrachtzeit festgelegt wird. Das führt zu folgender Definition:

- Ist $\alpha=\operatorname{Pres}(\beta), g(\alpha, \mathrm{t} 1, \mathrm{t} 2, \mathrm{t} 3)=1 \mathrm{gdw} . \mathrm{t} 3{ }^{*} \mathrm{t} 2$, und wenn $g(\beta, \mathrm{t} 1, \mathrm{t} 2, \mathrm{t} 3)=1$; ist $\beta \in \mathrm{SB}$, so ist dies der Fall, wenn $f(\beta, \mathrm{t} 3)=1$.

8 Die folgende Analyse folgt Ballweg 1988, Kap. 1-3. 
Das bedeutet, daß ein Satz im Präsens dann wahr wird, wenn das eingebettete Satzradikal an der Evaluationszeit wahr wird. Diese sehr liberale Analyse mag zunächst kontraintuitiv aussehen, aber man beachte, daß die Betrachtzeit in jedem Modell $\mathfrak{R}$ fest vorgegeben ist. Schließlich haben wir noch temporale Adverbialia zur Verfügung, die wir zur Spezifizierung von Betrachtzeiten benutzen, und auch mit Hilfe Grice'scher Räsonnements können wir Informationen über Betrachtzeiten ableiten, wie z.B. für (1), wo der zeitlose Charakter der Bedeutung des Satzradikals eine Spezifizierung der Betrachtzeit überflüssig macht. Auch der vorangehende Kontext kann Hinweise liefern; dies ist ein erster Hinweis darauf, daß eine dynamische Analyse wünschenswert ist. ${ }^{9}$

\section{Praeteritum}

Die Wahrheitsbedingungen für Pret können einfach dahingehend formuliert werden, daß postuliert wird, daß die Betrachtzeit vor der Sprechzeit liegt und sich die Evaluationszeit damit überlappt:

- Ist $\alpha=\operatorname{Pret}(\beta)$, so ist $g(\alpha, \mathrm{t} 1, \mathrm{t} 2, \mathrm{t} 3) 1 \mathrm{gdw} . \mathrm{t} 2<\mathrm{t} 1, \mathrm{t} 3 * \mathrm{t} 2$, und $g(\beta, \mathrm{t} 1, \mathrm{t} 2, \mathrm{t} 3)=1$.

Dies genügt zur Beschreibung einfacher Sätze im Präteritum, z. B

(4) Ludo durchstieg die Karlspitz-Ostwand,

aber schon bei Caesars

(5) Ich kam, ich sah und ich siegte.

gibt es Schwierigkeiten, da die Reihenfolge der drei Ereignisse nicht erfaßt ist. Auch hier scheint eine dynamische Analyse wünschenswert.

\section{Futur}

Wir ersparen uns eine Analyse des Futurs, da sie wegen seiner modalen Obertöne eine erhebliche Erweiterung des semantischen Apparates mit sich brächte:

- Die Funktion $f$ (,die Welt“ ) müßte für jedes Modell $\mathfrak{R}$ ersetzt werden durch eine Menge $F$ von solchen Funktionen ("möglichen Welten");

9 Vgl. Ballweg 1988. Kap. 4.3. Zur Einbezichung der Pragmatik sehe man Heringer 1983, sowie Grewendorf 1984, Ballweg 1984 und Lenerz 1986, sowie Vennemann 1987. 
- der Menge der möglichen Welten müßte eine Baumstruktur aufgeprägt werden, eine Struktur, die den "information-sets" à la Luce \& Raiffa entspräche, müßte definiert werden. Erst dann könnte man adäquate Wahrheitsbedingungen für das deutsche Futur formulieren, die seinem gleichzeitig temporalen und modalen Charakter gerecht würden. All dies würde hier zu weit führen. ${ }^{10}$

\section{(Praesens)-Perfekt}

In den Kommentaren zur Syntax haben wir eine kompositionale Behandlung des deutschen Praesens-Perfekts versprochen. Um dieses Versprechen einzulösen, müssen wir noch die Semantik des Perf-Operators angeben, der für Satzradikale im Infinitiv Perfekt steht und dann zeigen, wie sich die Bedeutung des PraesensPerfekts aus der Interaktion von Pres und Perf ergibt.

Zunächst einige empirische Bemerkungen: Das Problem der Beschreibung des deutschen Praesens-Perfekt liegt vor allem darin begründet, daß es Beispiele gibt, wo es vom Praeteritum semantisch fast nicht unterschieden ist, $\mathbf{z}$. B.:

(6a) Wir waren gestern im Kino.

(6b) Wir sind gestern im Kino gewesen.

In beiden Sätzen laufen die Intuitionen von Sprechern des Deutschen darauf hinaus, daß das Ereignis unseres Kinobesuches in der durch gestern gegebenen Betrachtzeit liegt. Eine weitere Komplikation liegt darin, daß für Sprecher oberdeutscher Varietäten ohnehin, zumindest in gesprochener Sprache, nur (6b) in Frage kommt, da sie Praeteritumsformen allenfalls bei kurzen, häufig gebrauchten Verben verwenden, wie $z$. B. bei sein und Modalverben.

Andererseits gibt es Fälle, in denen Sprecher, die über beide Formen verfügen, klar unterscheiden, insbesondere in telischen Sätzen mit temporalen Adverbialen wie:

(7a) Jetzt hat der Schiedsrichter dem VIB einen Freistoß zugesprochen.

wohingegen kaum jemand

?(7b) Jetzt sprach der Schiedrichter dem VIB einen Freistoß zu.

${ }^{10} \mathrm{Vgl}$. Ballweg 1988 und 1989. 
akzeptieren würde. ${ }^{11}$ Man betrachte auch

(8a) Viel wichtiger ist jetzt aber der Export von Kupfererz geworden.

versus

(8b) Viel wichtiger wurde jetzt aber der Export von Kupfererz.

Das temporale Adverb jetzt ist ein guter Indikator, da es sich in (8b) nicht auf die Sprechzeit beziehen kann, in (8a) aber sehr wohl. Dies unterstützt unsere kompositionale Behandlung, denn wenn Perfektsätze als $\operatorname{Pres}(\operatorname{Perf}(\alpha))$ formalisiert werden, können wir jetzt so placieren, daß es sich bei der Interpretation auf die Sprechzeit bezieht. Sätze wie

(9a) Gestern hat Reinhold den Cho Oyu bestiegen.

(9b) Gestern hat Reinhold alle 14 Achttausender bestiegen.

zeigen, daß die Skopusambiguitäten bei temporalen Adverbialia, die unsere kompositionale Analyse voraussagt, gut zu der Beobachtung passen, daß bei den bevorzugten Lesarten in (9a) das Ereignis im durch gestern gegebenen Betrachtzeitintervall lokalisiert wird, in (9b) jedoch davor. Die Formalisierungen

Pres(gestern(Perf( $\alpha)))$ und Pres $(\operatorname{Perf}(\operatorname{gestern}(\alpha)))$

geben diese Intuitionen auf natürliche Weise wieder.

Bisher haben wir mehr oder weniger implizit temporale Adverbialia als Spezifikation der Betrachtzeit behandelt. Um diese Strategie angesichts der Beispiele (7) - (9) beibehalten zu können, müssen wir für Sätze im Praesens-Perfekt zwei Betrachtzeiten annehmen, nämlich eine für den Praesens- und eine für den Perfektbestandteil. Am besten läßt sich das dadurch bewerkstelligen, daß wir den Perfektoperator so analysieren, daß er von der "alten" Betrachtzeit her eine neue einführt.

Dies ergibt insofern eine neue Perspektive, als das erste Zeitintervall in den Tripeln für unsere Interpretation nicht mehr als Sprechzeit gedeutet wird, sondern als Ursprungszeit ${ }^{12}$, von der her die Betrachtzeit gewonnen wird. Sprechzeit wäre in dieser Sicht nur ein Spezialfall von Ursprungszeit, nämlich die Ur-Ursprungszeit. Dies führt zu folgender Definition der Interpretationsklausel für Perf:

$"$ Es bleibt dem Jubilar überlassen, ob es sich hier um den VfB Stuttgart oder den VfB Leipzig handelt.

12 Bei Åqvist/Guenthner 1978: "time from where". 
- Ist $\alpha=\operatorname{Perf}(\beta), g(\alpha, \mathrm{t} 1, \mathrm{t} 2, \mathrm{t} 3)=1 \mathrm{gdw} \cdot g(\beta, \mathrm{t} 2, \mathrm{t} 4, \mathrm{t} 5)=1$, wobei $\mathrm{t} 4<\mathrm{t} 2$ und $\mathrm{t} 5 * \mathrm{t} 4$.

$\mathrm{t} 1$ und $\mathrm{t} 2$ sind die Ursprungs- bzw. Betrachtzeit für die Interpretation des übergeordneten Tempusoperators Pres, Pret oder Fut; die "alte" Betrachtzeit t 2 dient als Ursprungszeit für die Einführung der "neuen" Betrachtzeit 14 . Diese Definition scheint eine adäquate Semantik für die kompositionale Behandlung der zusammengesetzten Zeiten zu geben, die in der Syntax von ADETAL vorgesehen ist. Aber bei genauerem Hinsehen gibt es einige Probleme:

Der für die Interpretation von Perf vorgesehene Modellwechsel macht es nötig, statt eines einzigen Modells $\mathfrak{R}$ mehrere zur Verfügung zu haben, genauer gesagt eine Familie von dreifach indizierten Modellen, wobei die drei Indices Zeitintervalle sind:

$\Re_{t x, t y, t z \in T}$.

Wenn wir diese zur Verfügung haben, zeigt sich, daß die Interpretation für Perf zu stark formuliert ist, wenn sie ein Modell als neuen Interpretationskontext bereitstellt. Vielmehr scheint es klug zu sein, die Interpretation so zu formulieren, daß Perf eine Menge von geeigneten Modellen aus $\mathfrak{N}_{\mathrm{tx}, \mathrm{ty}, \mathrm{tz} \in \mathrm{T}}$ auswählt. ${ }^{13}$

Die Idee des Modellwechsels, wie wir sie für die Interpretation von Perf formuliert haben, scheint die Möglichkeit zu bieten, auch die oben erwähnten Probleme bei der Interpretation der finiten Tempora zu lösen. Versuchen wir also, eine dynamische Interpretation für Adetal zu formulieren! Die Analyse der temporalen Adverbialia stellen wir zurück und nehmen sie dann im Rahmen der neuen Interpretation vor.

\subsection{Eine dynamische Interpretation für ADETAL}

The future now will later be past, for the times, they are a-changin'

Bei der Entwicklung einer dynamischen Interpretation wollen wir die Ideen, die wir bei der Definition des Perfektoperators benutzt haben, weiterentwickeln. Dabei folgen wir dem Vorbild der dynamischen Prädikatenlogik von Groenendijk

${ }^{13}$ Eine Alternative bestünde in der Konstruktion von partiellen Modellen; jedes solche Modell definiert eine Menge von Modellen, seine Extensionen. Siehe Kamp/Reyle 1993, S. 92-98. 
und Stokhof ${ }^{14}$ und interpretieren Sätze an Mengen von Paaren von Modellen, wobei in jedem Paar das zweite Modell sich vom ersten höchstens in der Wahl der drei designierten Zeitintervalle unterscheiden darf.

Sei $\mathfrak{R}_{t x, t y, t z \in T}$ eine Familie von dreifach indizierten Modellen dergestalt, daß jedes Element von $\mathfrak{R}$ wie oben definiert ist.

Ein $\Re$, bei dem tx, ty, $\mathrm{tz}=\mathrm{t} 0, \mathrm{t} 0, \mathrm{tz}{ }^{*} \mathrm{t} 0$ gilt, heiße "Urmodell".

Eine Interpretation I sei eine Funktion von $S B \times \mathfrak{R}_{\mathrm{ta}}$, tb, tc $\in \mathrm{T} \times \mathfrak{R}_{\mathrm{td}}$, tc, if $\in \mathrm{T}$ nach $\{0,1\}$, so daß gilt:

Ist $\alpha \in$ SB oder ist $\alpha=\neg(\beta)$, oder ist $\alpha=(\beta \wedge \gamma)$, so ist $\mathrm{I}\left(\alpha,\left\{\left\langle\mathfrak{R}, \mathfrak{R}^{\prime}>\right\}\right)=1\right.$ gdw. $\mathfrak{R}_{\mathrm{ta}, \mathrm{tb}, \mathrm{tc} \in \mathrm{T}}=\mathfrak{R}_{\mathrm{td}, \mathrm{tc}, \mathrm{tf} \in \mathrm{T}}$ und $g(\beta, \mathrm{ta}, \mathrm{tb}, \mathrm{tc})=1$.

Dies heißt einfach, daß die Interpretation von nicht temporalisierten Formeln und Junktoren die drei Intervalle unverändert läßt.

Ist $\alpha=\beta(\gamma), \beta \in$ TO $\cup$ TADV, $\gamma \in$ WFF, so $\mathrm{I}\left(\alpha, \mathfrak{R}_{\text {ta,tb.tc }}\left|\mathrm{C}_{\beta}\right| \mathfrak{R}_{\mathrm{ta}, \mathrm{tb}, \mathrm{tc}}\right)=1$ gdw. $g(\gamma, t d$, te, tf $)=1$ für mindestens ein Modell $\mathfrak{R}$, das durch $C_{\beta}$, die Übergangsbedingung des entsprechenden Tempusoperators oder Temporaladverbials $\beta$, aus $\mathfrak{R}$ abgeleitet ist.

In diesem Rahmen müssen wir nun für jeden einzelnen Tempusoperator nur noch die spezifische Übergangsbedingung $C$ spezifizieren:

$\mathrm{C}_{\text {Pres }}$ :

a) Ist in $\mathfrak{R} g(\beta$, ta, tb, tc $)=1$, so ist $\mathfrak{R}=\mathfrak{R}$,

b) wenn nicht, und wenn es ein $\mathrm{tf}^{*}$ tb gibt, so daß tc $<\mathrm{tf}$, und $g(\beta, \mathrm{ta}, \mathrm{tb}, \mathrm{tf})=$ 1 , dann ist $\mathfrak{R}=\mathfrak{R}^{\prime}$ mit dem einzigen Unterschied, daß tc durch tf ersetzt wird. c) ist weder a) noch b) der Fall, so ist $t d=t 0, t e=t \#$ und $t f^{*}$ te.$(t \#$ ist das salienteste Intervall im Kontext, das sich von tb unterscheidet, und $t \#=t 0$, wenn kein anderes salientestes Intervall vorhanden ist).

Diese Bedingungen laufen darauf hinaus, daß die Interpretation für das Praesens entweder das „alte“ Modell beibehält, falls der ein gebettete Satz dort wahr ist, oder daß eine neue Betrachtzeit gewählt wird aus dem Kontext, im Zweifelsfall die Sprechzeit. Betrachtet man die Sätze

${ }^{14}$ Groenendijk/Stokhof 1991. 
(10a) Im 8. Jahrhundert leben Franken am Niederrhein.

(10b) Ausgrabungen beweisen das.

so erkennt man die Gründe für diese Festlegung: in (a) bleibt die Interpretation auf der durch im 8. Jahrhundert spezifizierten Betrachtzeit, in (b) springt sie zurück auf t0. Das folgende Satzpaar

(11a) Im ersten Jahrhundert kommen die Römer nach Bayern.

(11b) Sie bleiben dort lange.

illustriert, warum es nötig ist, bei festgehaltener Betrachtzeit die Evaluationszeit weiterbewegen zu können, nämlich um die Reihenfolge mehrerer Ereignisse wiedergeben zu können. Die Option, das im Kontext salienteste Intervall auszuwählen, ist in Beispielen am Werk wie

(12a) Es ist Sonntagmorgen.

(12b) Paul hat gestern viel gearbeitet.

(12c) Jetzt liest er die Zeitung.

wo (c) die „alte“ Betrachtzeit von (a) wieder aufgreift.

Die Ordnung der Optionen ist als Praeferenzordnung zu verstehen, wodurch eine Art Trägheitsprinzip zum Ausdruck kommt: „Halte die Interpretationsintervalle fest, solange es geht!“

Cret:

a) Ist tb $<\mathrm{t} 0$ und $g(\beta, \mathrm{ta}, \mathrm{tb}, \mathrm{tc})=1$, so ist $\mathfrak{R}=\mathfrak{R}$.

b) Wenn nicht, und wenn tb $<$ t0, und es gibt ein tf ${ }^{*}$ tb und tc $<$ tf,und $g(\beta$, ta, tb, tf ) $=1$, so ist $\mathfrak{R}=\mathfrak{R}^{\prime}$ mit dem einzigen Unterschied, daß tc durch tf ersetzt wird.

c) Ist weder a) noch b) der Fall, so ist td = t0; te wird so gewählt, daß te $<$ td und wenn möglich te $=\mathrm{t} \#$ und $\mathrm{tf}^{*}$ te.

Auch hier ist die Strategie wieder: Ist der eingebettete Satz im im Kontext erreichten Modell wahr, so wird dies beibehalten (siehe (12) unten), wenn nicht, so wird die Ursprungszeit und die Betrachtzeit festgehalten und nur die Evaluationszeit weiterbewegt (siehe (13) unten), und wenn diese beiden Optionen versagen, so wird ein neues Betrachtzeitintervall te vor t0 gewählt, wenn möglich das im Kontext salienteste Intervall $\mathbf{t}$ (14c); wenn nicht, so wird te arbiträr gewählt (wie in (14a), als Beginn eines Textes aufgefaßt). 
(12) 1618 brach der dreißigjährige Kricg aus.

(13) Ich kam, ich sah und ich siegte.

(14a) Es war ein Sonntagmorgen.

(14b) Paul hatte gestern viel gearbeitet.

(14c) Jetzt las er die Zeitung.

Der wichtige Unterschied zum Praesens mit vergangenen Betrachtzeiten ist, daß das Präteritum eine neue Betrachtzeit einführen kann, wohingegen das Praesens nur eine im Kontext schon vorhandene aufgreifen darf. Somit läßt sich das Praesens, das in der Reichenbachianischen Semantik als eine Art Passepartout-Tempus erschien, in der dynamischen Interpretation adäquat als kontextdependentes Tempus beschrieben, das im Zweifelsfall die Sprechzeit als Betrachtzeit hat. Die Übergangsbedingung $C$ für Perf, dessen Analyse ja schon in der Reichenbachianischen Semantik quasi dynamisch war, kann jetzt folgendermaßen formuliert werden:

$C_{\text {Perf : }}-t d=t b, t e<t b, t f * t e$

Der Perf-Operator ändert also immer den vom Obertempus übernommenen Kontext.

\section{Temporaladverbialia}

Bevor wir zeigen können, wie die semantische Maschinerie bei der Analyse eines kurzen Textstückes arbeitet, müssen wir zunächst noch die Analyse einiger temporaler Adverbialia nachholen:

$$
\mathrm{C}_{\mathrm{da}}: \mathrm{td}=\mathrm{tc}, \mathrm{td} \subseteq \mathrm{te}, \mathrm{td}-\mathrm{te} \text { und } \mathrm{tf} * \mathrm{te}
$$

Das läuft darauf hinaus, daß die Übergangsbedingung für $d a$ die alte Evaluationszeit als Ursprungszeit übernimmt, die alte Evaluationszeit in der neuen liegt und deren Beginn vorgibt und eine neue Evaluationszeit eingeführt wird.

$$
\begin{aligned}
& \text { dann } \\
& \mathrm{C}_{\text {dann }}: \mathrm{td}=\mathrm{tc}, \mathrm{td}<\mathrm{te}
\end{aligned}
$$

dann hat also als Übergangsbedingung, daß die alte Evaluationszeit als Urspungszeit dient, von der aus eine neue, danach liegende Betrachtzeit eingeführt wird. Dies erklärt den Bedeutungsunterschied zwischen:

(15a) Günther studierte Logik. Da war er glücklich.

(15b) Günther studierte Logik. Dann war er glücklich. 
bis

$C_{\text {bis }}: t d=t c, t d<t e, t e *$ tf und $t d \cup$ te und damit auch td $\cup$ tf $\in \mathrm{T}$ (d.h. sie sind konvex, bilden ein zusammenhăngendes Intervall).

Das läuft darauf hinaus, daß das im mit bis eingeleiteten Untersatz dargestellte Ereignis unmittelbar nach dem im Obersatz geschilderten Ereignis eingeordnet wird, daß das Ereignisintervall für den Obersatz durch den Anfang des Ereignisintervalls des Untersatzes rechts begrenzt wird ${ }^{15}$, wie (16) zeigt :

(16) Ich ging immer, wenn ich vom Training kam, ganz nahe an Marie heran, bis ich mich in ihren Augen sah.

(Böll, Clown, S. 175.)

Hier begrenzt der Beginn des Ereignisintervalls von in die Augen sehen das Ereignisintervall von ganz nahe herangehen. Man beachte jedoch, insbesondere bei transformativen Verben, daß bei zusammengesetzten Tempora das entsprechende Ereignis der dem perfektivierten Satzradikal entsprechende Zustand ist! So ist in:

(17a) Später ging ich noch für Marie zur Apothcke. holtc ihr ein Schlafmittel und saß an ihrem Bett, bis sie eingeschlafen war.

(Böll, Clown, S. 149).

nicht das Ereignisintervall des Einschlafens rechte Begrenzung für das Ereignisinter-vall des Wartens, sondern das Ereignis des Eingeschlafenseins, d.h. des Schlafens, das somit als unmittelbarer Nachzustand des Einschlafens sowohl das Warten als auch das Einschlafen rechts begrenzt. Die erklärt auch, daß (17b) mit (17a) wahrheitskonditional äquivalent ist:

(17b) Später ging ich noch für Marie zur Apotheke. holte ihr ein Schlafmittel und saß an ihrem Bett, bis sie schlief.

${ }^{15}$ Man beachte, daß td $\cup$ te unter der Voraussctzung td $>$ te nur dann konvex sein kann, wenn entweder $t d$ ein rechts geschlossenes Intervall $\left[t_{x}: t_{y}\right]$ oder $\left.\left.t_{x}\right] ; t_{y}\right]$ und te cin links offenes Intervall $\left.t_{y}\right] ; t_{z}$ ] ist, oder wenn td rechts offen und te links geschlossen ist. Das von a und $b$ bestimmte geschlossene Intervall wird definiert als: $[a ; b]=\{x \mid a \leq x \leq b\}$, d.h. a und b gehören zum Intervall; das entsprechende offene Intervall als: $] a ; b=\{x \mid a<x<b\}$, d.h. a und $b$ gehören nicht zum Intervall. 
Nunmehr sind wir in der Lage, die Analyse eines Textstücks mit Hilfe der bisher entwickelten dynamischen Zeitlogik zu versuchen:

So fuhr ich also mit dem Wagen nach der Stadt (1). Auf halbem Wege kommt mir 'n anderer Wagen entgegen (2), und natürlich sitzt kein andrer als Tom Sawyer drin (3). Ich hielt an (4) und wartete (5), bis er rankam (6). Dann ruf ich (7): Stillgestanden ! Da hält er auch an (8). ${ }^{17}$

Der Einfachheit und Übersichtlichkeit halber werden wir statt der Mengen von Paaren von Modellen jeweils lediglich die drei designierten Intervalle angeben, die sich durch die Übergangsbedingungen für Tempora und temporale Adverbialia ergeben.Bei dem Text handelt es sich um den Anfang des 32. Kapitels; für den Kapitelanfang können wir ein Urmodell $\mathrm{t} 0, \mathrm{t} 0, \mathrm{tf}{ }^{*} \mathrm{t} 0$ annehmen.

Die Übergangsbedingung für das Präteritum in So fuhr ich ... führt zu einer Menge von Modellen, die durch folgende Struktur der designierten Intervalle gekennzeichnet sind:

$\mathrm{t} 0, \mathrm{t} 1, \mathrm{t} 2$ mit $\mathrm{t} 1<\mathrm{t} 0$ und $\mathrm{t} 2 * \mathrm{t} 1$.

Die Übergangsbedingung für das Präsens in Auf halbem Wege kommt ... kann die Betrachtzeit $\mathrm{t} 1$ festhalten und eine neue Evaluationszeit einführen, $\mathrm{t} 3$, so daß $\mathrm{t} 2<$ $\mathrm{t} 3$ und $\mathrm{t} 3 * \mathrm{t} 1$. Damit ergibt sich eine Menge von Modellen, die durch folgende Struktur der designierten Intervalle gekennzeichnet sind:

$\mathrm{t} 0, \mathrm{t} 1, \mathrm{t} 3$ mit $\mathrm{t} 1<\mathrm{t} 0, \mathrm{t} 2<\mathrm{t} 3$ und $\mathrm{t} 3 * \mathrm{t} 1$.

Man beachte, daß die hier plausible Weiterbewegung des Evaluationsintervalls nicht durch die Übergangsbedingung für das Tempus erzwungen wurde, sondern daß diese lediglich liberal genug ist, Raum für ein pragmatisches Weiterbewegen durch ein Ikonieprinzip zuzulassen.

Die Interpretation des Präsens in ... und natïrlich sitzt kein andrer als Tom Sawyer drin ... läßt alles beim alten, illustriert also das oben angesprochene Trägheitsprinzip; somit haben wir immer noch

$\mathrm{t} 0, \mathrm{t} 1, \mathrm{t} 3$ mit $\mathrm{t} 1<\mathrm{t} 0, \mathrm{t} 2<\mathrm{t} 3$ und $\mathrm{t} 3 * \mathrm{t} 1$.

${ }^{16}$ Eine Vorform der Analyse dieses Textes findet sich bereits in Ballweg 1991.

${ }^{17}$ Eine Alternative wăre, den am Schluß des vorherigen Kapitels erreichten Kontext zu nehmen, 1989, Ehrich/Vater 1988, Fabricius-Hansen 1986 und 1991, sowie Thieroff/Ballweg 1994. 
Die Interpretation für das Präteritum in Ich hielt an ... kann sowohl die Ursprungs- als auch die Betrachtzeit festhalten und lediglich eine neue Evaluationszeit $\mathbf{t} 4$ einführen, die nach $\mathrm{t} 3$ liegt . Damit ergibt sich

$\mathrm{t} 0, \mathrm{t} 1, \mathrm{t} 4$ mit $\mathrm{t} 1<\mathrm{t} 0, \mathrm{t} 3<\mathrm{t} 4$ und $\mathrm{t} 4 * \mathrm{t} 1$.

Die Interpretation für das Präteritum in ...ınd wartete ... führt ebenfalls nur eine neue Evaluationszeit ein, somit ergibt sich

$\mathrm{t} 0, \mathrm{t} 1, \mathrm{t} 5$ mit $\mathrm{t} 1<\mathrm{t} 0, \mathrm{t} 4<\mathrm{t} 5$ und $\mathrm{t} 5 * \mathrm{t} 1$.

Die Übergangsbedingung für bis nimmt die letzte Evaluationszeit als Ursprungszeit und führt je eine direkt anschließende Betracht- und Evaluationszeitzeit ein; so ergibt sich

$\mathrm{t} 5, \mathrm{t} 6, \mathrm{t} 7$ mit $\mathrm{t} 5<\mathrm{t} 0, \mathrm{t} 5<\mathrm{t} 6, \mathrm{t} 6 * \mathrm{t} 7$, und $\mathrm{t} 5 \cup \mathrm{t} 6, \mathrm{t} 5 \cup \mathrm{t} 7 \in \mathrm{T}$

Die Interpretation für das Präteritum rankam kann diesen für Präteritum geeigneten Kontext übernehmen.

Die Übergangsbedingung für damn ... nimmt die alte Evaluationszeit als Ursprungszeit und führt eine vollständig danach liegende neue Betrachtzeit ein. Das führt zu:

$\mathrm{t} 7, \mathrm{t} 8, \mathrm{t} 9$ mit $\mathrm{t} 7<\mathrm{t} 8$ und $\mathrm{t} 8 * \mathrm{t} 9$.

Die Interpretationsbedingung für ... ruf $i c h$... kann den durch die Interpretationsbedingung von dann erreichten letzten Kontext festhalten, und wir haben immer noch:

$\mathrm{t} 7, \mathrm{t} 8, \mathrm{t} 9$ mit $\mathrm{t} 7<\mathrm{t} 8$ und $\mathrm{t} 8 * \mathrm{t} 9$.

$\mathrm{C}_{\mathrm{da}}: \mathrm{td}=\mathrm{tc}, \mathrm{td} \subseteq \mathrm{te}, \mathrm{td}$-te und $\mathrm{tf} \subseteq$ te.

Die Übergangsbedingung für $d a$... nimmt die alte Evaluationszeit als Ursprungszeit, führt eine neue Betrachtzeit ein, die die Ursprungszeit (= alte Evaluationszeit) enthält und gleichzeitig mit ihr beginnt und führt eine neue Evaluationszeit ein, die darin liegt ( und damit nicht vor der alten Evaluationszeit beginnt!). Damit erhalten wir:

$\mathrm{t} 9, \mathrm{t} 10, \mathrm{t} 11$, mit $\mathrm{t} 9 \subseteq \mathrm{t} 10, \mathrm{t} 9-\mathrm{t} 10$ und $\mathrm{t} 11 \subseteq \mathrm{t} 10$.

Die Übergangsbedingung für das Präsens in ... hält er aıch an ... kann diesen Kontext beibehalten und wir bleiben bei:

$\mathrm{t} 9, \mathrm{t} 10, \mathrm{t} 11$, mit $\mathrm{t} 9 \subseteq \mathrm{t} 10, \mathrm{t} 9-\mathrm{t} 10$ und $\mathrm{t} 11 \subseteq \mathrm{t} 10$. 
Eine graphische Übersicht dieser Interpretationsskizze soll nochmals verdeutlichen, daß die Reihenfolge der Ereignisse durch die vorgeschlagenen Interpretationsregeln intuitiv befriedigend dargestellt wird:

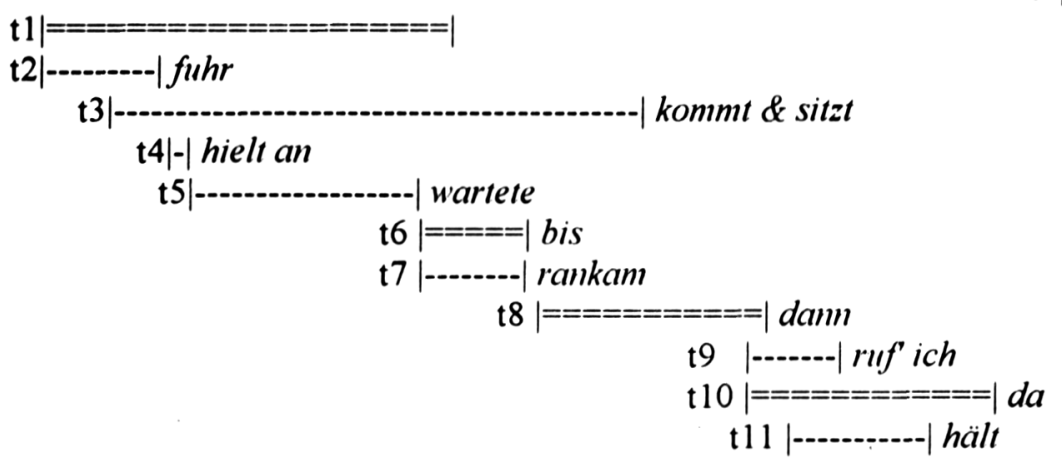

|=====| bezeichnet Betrachtzeiten, |---------| bezeichnet Evaluationszeiten.

\section{Schlußbemerkungen}

Die Analyse und die Übersicht zeigen zunächst, daß die berichteten Ereignisse durch die vorgeschlagenen Interpretationsregeln für Tempora und temporale Adverbialia so geordnet werden, wie es der Intuition eines Lesers entspricht. Die semantischen Regeln bedürfen dabei der Ergänzung durch pragmatische Prinzipien, wo die Interpretation gerade der finiten Tempora mehrere Optionen bereitstellt.

Die hier vorgeschlagene Analyse knüpft unmittelbar an von Reichenbach beeinflusste Tempusanalysen an, was angesichts der starken Verbreitung solcher Analysen sicher vorteilhaft ist. 


\section{Literatur}

Abraham,W./Janssen, T. (Hrsg.) (1989): Tempus - Aspekt - Modus. Die lexikalischen und grammatischen Formen in den germanischen Sprachen. Tübingen: Niemeyer (Linguistische Arbeiten 237).

Åqvist, L. (1976): Formal semantics for verb tenses as analyzed by Reichenbach. In: Teun A. van Dijk, (Hrsg.) 229-236.

Åqvist, L./Guenthner, F. (1978): Fundamentals of a theory of verb aspect and events within the setting of an improved tense logic. In: Guenthner/Rohrer (Hrsg.) 167-199.

Askedal, J. O. et al. (Hrsg.) (1983): Festschrift für Laurits Saltveit. Oslo: Universitetsfoerlaget 1983.

Ballweg, J. (1984): Praesentia non sunt multiplicanda praeter necessitatem.In: Stickel, Gerhard (Hrsg.) 243-261.

- (1988): Die Semantik der deutschen Tempusformen. Düsseldorf: Schwann (Sprache der Gegenwart 70).

- (1991): German tenses, tense logics, context change and the temporal interpretation of texts. In: Gvozdanovic/Janssen (Hrsg.) 17-34.

Băuerle, R./Egli, U. und v. Stechow, A. (Hrsg.) (1979): Semantics from different points of view. Berlin, Heidelberg, New York: Springer.

Ehrich, V./Vater, H. (Hrsg.) (1989): Temporalscmantik. Beiträge zur Linguistik der Zeitreferenz. Tübingen: Niemeyer (Linguistische Arbeiten 201).

Fabricius-Hansen, C.(1986): Tempus fugit. Düsscldorf: Schwann (Sprache der Gegenwart 64).

Grewendorf, G. (1984): Besitzt die deutsche Sprache cin Präsens? In: Stickel (Hrsg.) 224-242.

Groenendijk, J./Stokhof, M. (1991): Dynamic Predicatc Logic. Linguistics and Philosophy 14, 39-100.

Guenthner, F./Rohrer, C. (Hrsg.) (1978): Studies in Formal Semantics, Amsterdam, New York, Oxford: North Holland 1978.

Gvozdanovic, J./T. Janssen (Hrsg.) (1991): The function of tense in texts. Amsterdam/Oxford/New York/Tokyo: North Holland.

Heringer, H. J. (1983): Präsens für die Zukunft. In: Askedal, J. O. et al. (Hrsg.) 110-126.

Kamp, H. (1979): Events, instants and temporal reference. In: Bäuerle/Egli und v. Stechow, (Hrsg.) (1979), 376-417.

Kamp, H./Reyle, U. (1993): From Discourse to Logic. 2 Bde. Dordrecht. Boston. London: Kluwer.

Krifka, M. (1986): Nominalreferenz und Zcitkonstitution. München: Fink .

Lenerz, J. (1986): Tempus und Pragmatik - oder: Was man mit Grice so alles machen kann. LB $102,1984,136-154$.

Lơbner, S. (1988): Ansätze zu einer integralen semantischen Theorie von Tempus, Aspekt und Aktionsarten. In: Ehrich/Vater (Hrsg.).

Reichenbach, H. (1947): Elements of symbolic Logic. New York: Free Press.

Rohrer, C. (Hrsg.) (1977): On the logical analysis of tense and aspect. Tübingen: Niemeyer (Linguistische Arbeiten 83).

Smith, C. (1977): Temporal structure in discourse. In: Rohrer (Hrsg.). 355-374.

Stickel, G. (Hrsg.) (1984): Pragmatik in der Grammatik. Düsseldorf: Schwann (Sprache der Gegenwart 60).

Thieroff, R./Ballweg, J. (Hrsg.) (1994): Tense Systems in European Languages. Tübingen: Niemeyer (Linguistische Arbciten 308).

Twain, M. (1953): Hucklebcrry Finn. Deutsch von Ulrich Johan sen, 2. Aufl. München: Droemer/Knaur.

van Benthem, J. (1980): Points and Periods.In: Rohrer. Chr. (Hrsg.) 39-58. 
van Dijk, T. (1976): Pragmatics of Language and Literature. Amsterdam. New York, Oxford: North Holland.

Vennemann, T. (1987): Tempora und Zeitrelation im Standarddeutschen. Sprachwissenschaft Bd. 12, 2, 234-249.

von Stechow, A./Wunderlich, D. (Hrsg.) (1991): Semantik - Ein internationales Handbuch der Zeitgenössischen Forschung. Berlin, New York: de Gruyter. 